\title{
Anti Collision and Secured Level Crossing System
}

\author{
K. Vidyasagar \\ Dept. of ECE, SSIT \\ Sathupally, T.S, India.
}

\author{
P. Sekhar Babu \\ Dept. of ECE, SSIT \\ Sathupally,T.S,.India.
}

\author{
R. RamPrasad \\ Dept.of ECE,SSIT \\ Sathupally,T.S,.India.
}

\begin{abstract}
The road and rail accidents are increasing with the advancement of human errors. Majority of the level crossing positions are not guided by any accident preventive mechanism and even not accompanied by a skilled person. This paper presents an automatic rail gate control system at level crossing positions and accident prevention mechanism. Two vibration sensors are used to control the open and close state of the gate at level crossing position. An ultrasonic sensor is positioned to detect an unauthorized object on the track. Open and Close status of the gate and unauthorized object on the track will be communicated with the central control room using wireless communication protocol. The experimental results proved the proposed mechanism is a prudent approach to safeguard the human and to curtail the train accidents.
\end{abstract}

\section{Keywords:}

Rail gate, Vibration sensor, ultrasonic sensor.

\section{INTRODUCTION}

Indian railways daily transporting 14 million passengers by 2 million kilometer rail track per day. Safety is of outstanding significance to Indian railways. Safety and reliability are closely linked components. Deterioration in the safety mechanism is preceded by the increase in the number of failures. Overlook the warning signals can be calamitous as each of these is an accident waiting to happen. A man machine system inherently may enhance the reliability of the equipment is the most significant factor in the safety of the rail transport system. The Proposed approach may exhibit near Zero level of failure rate. With the advancement of Technology it is imperative that the accidents have to be minimized. It is also imperative that the technology in use is kept in fine fettle. $20 \mathrm{~mm}$ Square rod was mounted on " $6 \mathrm{~mm}$ $\times 2$ inch" flat metal sheet used to develop the rail track. The model rail was fabricated using $20 \mathrm{~mm} \times 6$ inch flat round sheets shaped as train wheels. Two motor driven rail gates are equipped across the rail track.

\section{LITERATURE SURVEY}

Sandya Goutam et.al concentrated on predicting the major cause of railway accidents that is collision on the same track. For this purpose a technology used to identify train positions, collision detection as well as the points at where collisions may occur has been used. The primary goal of this paper is anti-collision system to identify such collision points and to report the error cases to main control room, nearby station as well as grid control stations. To build this system, advanced sensing technology, long distance communication system (RS 485protocol), microcontroller (8051) and wireless Communication protocol has been used. [1]

This paper presents a pressure sensor based swift response anti-collision system for an automatic railway gate control. By replacing the manual system of railway gate control at the level crossing it has been develop an automatic system in which the arrival and departure of the train will be sensed automatically to control the gate. Pressures switch which has been integrated in this anti-collision system for the railway. By chance if a vehicle gets stuck at the level crossing of the rail-line, the pressure sensor will detect the object and will take necessary action by following the developed algorithm. The whole operation of this project has been controlled by a Microcontroller PIC16F84A. Two IR sensors have been used to detect the arrival and departure of the train. IR sensor will give the signal to the Microcontroller and the Microcontroller will pass this signal to the DC motor and it will rotate as its requirement [2]. This work is concentrated on anti-collision system to identify the collision points and to report these error cases to main control room, nearby station as well as grid control stations. Implementation of an efficient Zig-Bee based Train Anti-Collision for railways is being proposed in this paper [3]. The paper has four sub modules namely, Train Module, Control Centre Module, Signaling Post Module and Level Crossing Gate Module. A safe distance of $1 \mathrm{Km}$ has been maintained between the trains after applying the emergency brake in case of collision detection. It is expected that if this system is implemented widely, train collisions and accidents at the Manned/Unmanned level crossing gate can also be avoided in the future [4]. This paper describes the controlling of the level crossing gate. one pair of transmitter and receiver is fixed at upside (from where the train comes) at a level higher than a human being in exact alignment and similarly the other pair is fixed at down side of the train direction. Sensors are fixed at $1 \mathrm{~km}$ on both sides of the gate [5]. The objective of this paper is to control the railway tracks by using anti-collision techniques. This model is implemented using sensor technique. Sensors are placed at a certain distance from the gate detects the approaching train and accordingly controls the operation of the gate. Also an indicator light has been provided to alert the motorists about the approaching train [6].This paper describes the rail gate control system using IR sensors the PIC 16F877A microcontroller is used as controller [7]. This work utilizes two powerful magnetic sensors is fixed at upside and similarly the other magnetic sensor is fixed at down side of the train direction. Sensors are fixed on both sides of the gate [8]. This paper discus the GPS and GSM technology equipped with train to curtail the train accidents [9]. This paper discusses several features which prevent train accidents. It includes automatic speed controlling in curves, collision detection, fire detection, detaching of couch automatically when fire is detected in it, automatic railway gate control and track continuity. This system makes use of IR sensors, fire sensor, Zig Bee and other embedded systems [10].This paper describes an intelligent railway crossing control system for multiple tracks that features a controller which receives messages from incoming and outgoing trains by sensors. These messages contain detail information including the direction and identity of a train. Depending on those messages the controller device decides whenever the railroad crossing gate will close or open[11]. 


\section{THE ORETICAL APPROACH}

\subsection{Vibration Sensor}

A piezoelectric transducer used as a vibration sensor. The displacement from the mechanical neutral axis generates strain within the piezoelectric element and generates voltage sinked output signals. The Maximum linear distance on the surface is estimated from the recommended detector location to rail gate point using the following procedure.

Compute $\mathrm{X} 1=\mathrm{h}+\mathrm{w}$

Compute $\mathrm{X} 2=2 \mathrm{~d}+\mathrm{w}$

Where $\quad h=$ Safe Height

$\mathrm{w}=$ Safe Width

$$
\mathrm{d}=\text { Safe Depth }
$$

Find $\mathrm{X}=$ minimum $(\mathrm{X} 1, \mathrm{X} 2)$

Compute $\mathrm{J}=\mathrm{w}+\mathrm{d}$

Find $\mathrm{D}=\operatorname{maximum}(\mathrm{X}, \mathrm{J})$

Where $\mathrm{D}=$ Maximum Linear Distance.

The Vibration is a periodic displacement of an object around a center static position. Vibration is measured as an acceleration or displacement. The sensor used is a non contact displacement sensor. The sensor is mounted with a small gap between the sensor probe and the rail track i.e. the surface of the vibrating object. The sensor outputs precise vibration signal. The total vibration number is determined by using the Total indicator reading (TIR) i.e. the deference between the maximum value of negative and positive measurements. The Difference between the maximum positive and maximum negative signal amplitude measurement is called as total vibration. The resultant output signal is transferred to the gate controller. The output of the controller is used to drive the gates. The open and close state of the gate depends on the signal strength. The zero signals keep the gate normally in closed state. The status of the gate information is transferred to the remotely located control room.

\subsection{Communication}

Low power short range communication is established between the rail gate control unit and the control room using IEEE 802.15.4. Offset Quadrature phase shift keyed modulation stream (OQPSK) with $2.4 \mathrm{G} \mathrm{Hz}$ is used for transmitting the information to the remote control room. The received signal is interfaced to personal computer using recommended standard 232-D serial communication interface device. Flash magic software is used for better operation. The targeted device i.e. the model train is interfaced to COM port using DB-9 connector. 9600 Baud rate is used for transmission. The necessary code is burned in code memory. The received status of the train, gate open close, and any unauthorized object on the track from the transmitter section is displayed at the control room. This paper also focused on controlling the train remotely. If any unexpected conditions received from the gate control circuit and even from the moving train, the appropriate control signals are transferred to the train from the control room using the flash magic software.

\subsection{Object Targeted Sensor}

An ultrasonic sensor LV-Prox Sonar-EZ is used for object detection in the detection zone. Multiple sensors are used to sense when any object enters the detection zone. The target will be released only after leaving the detection zone. The Sensor captures the target in just 2.5 seconds and releases the serial output signal. The clearance of the targeted object on the track will be sensed within 1.5 seconds by the sensor. The position of the track is transmitted to the personal computer using RS-232D.

\subsection{Controller}

AT 89S52 is used as a controller at two stages. One for Rail gate control section and other for train control section. Ultrasonic sensor LV-Prox Sonar-EZ is compatible to AT89S52 Micro controller. Interrupt 4 is used for serial communication. The Receiver Interrupt (RI) is used to control the train and to transfer the status to the control room using IEEE 802.15.4. Port 0 is configured as an output port and is used to drive the motors 1 and 2 . Port 0 is also used for manual operation of the train for start and stop position. Port 3 is configured for vibration sensors. The Microcontroller read the input port to know the status of the vibration sensor. If the sensor outputs a ' 5 ' Volts logic signal the gate motors will be energized. The open close state of the gates depends on the status of the signal at the input port 3. Ultra sonic sensor LVProx Sonar -EZ is interfaced to port ' 1 '. Port1 is configured as an input port. If the port ' 1 ' outputs a 5 Volts logic signal the microcontroller drives the motors to stop the train and an interrupt -4 causes to set the RI bit one. This interrupt cause to transmit the status information to remote control station and also displayed on LCD interfaced to the microcontroller.

\section{IMPLEMENTED ALGORITHM}

Step1: Initialize port ' $0 \_0$ ' and ' $0 \_1$ ' for train driving motor 1

Initialize port ' $0 \_5$ ' and ' $0 \_6$ ' for train driving motor 2

Step2: Initialize port ' $0 \_2$ and ' 0 3' for Switch 1 and Switch2 for start and stop the train respectively.

Step3: Initialize port '0 7' for Buzzer

Step4: Initialize Port 3_5 and port 3_6 for Vibration sensor 1 and 2.

Step5: Initialize port3_7 for vibration sensor 3.

Step6: Initialize port'1' for ultrasonic sensor

Step7: Read the target detection sensor (port1), if the logic level is low then go to next step else stop the train and transmit to the control room

Step8: Read the sensor1 (port3_5), if the signal logic level is ' 5 ' Volts then close the rail gates else open it.

Step9: Enable the Interrupt-4 to set $\mathrm{RI}=1$ and to transmit the status to the control room.

Step10: Insert step7.

Step11: Read the sensor 2, if the logic level is ' 5 'Volts then open the rail gates else close it.

Step12: Enable the output port to transmit to the control room

Step13: Insert step7.

Step14: Read the sensor 3, if the logic level is'5'Volts then stop the train (end Point) else move on the track.

Step15: Go to step1. 


\section{RESULTS AND ANALYSIS}

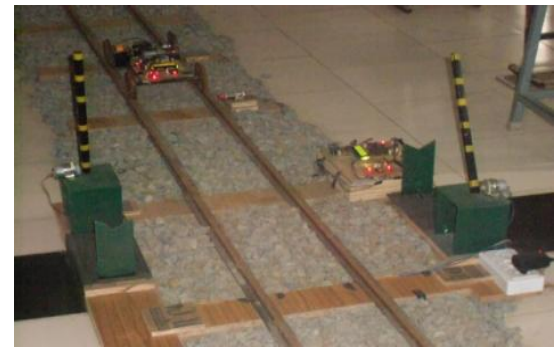

Figure 1: Open state of the rail gate

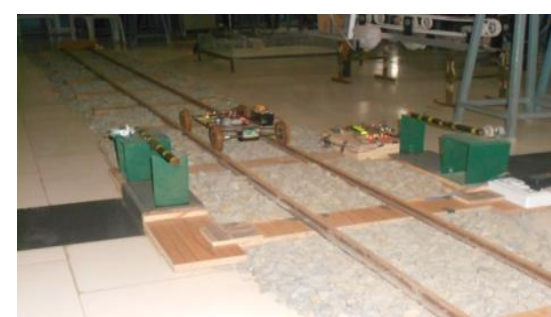

Figure 2: Close state of the rail gate

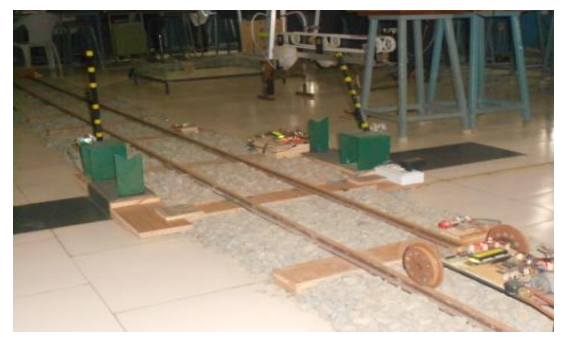

Figure 3: Train leaving the rail gate

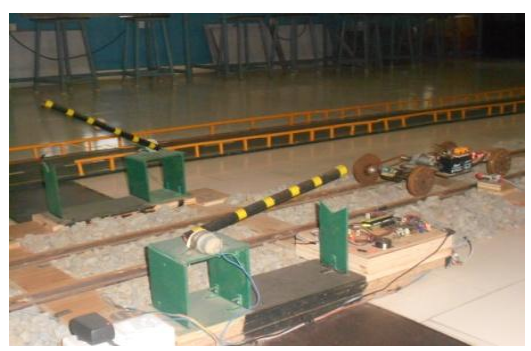

Figure 4: Train moving close to the gate

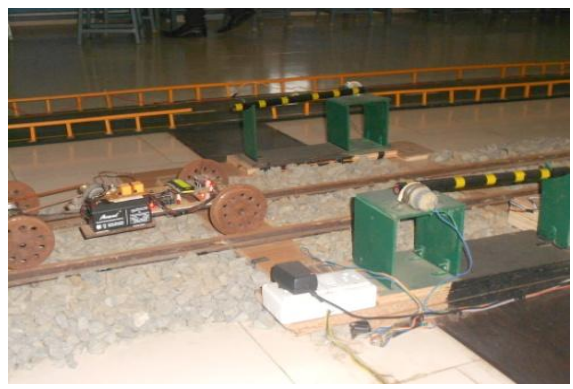

Figure 5: Train leaving the rail gate

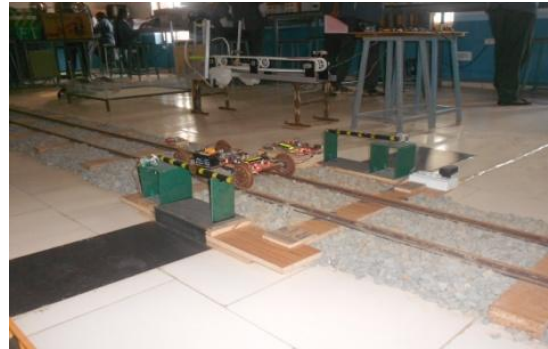

Figure 6: Gate fully closed state

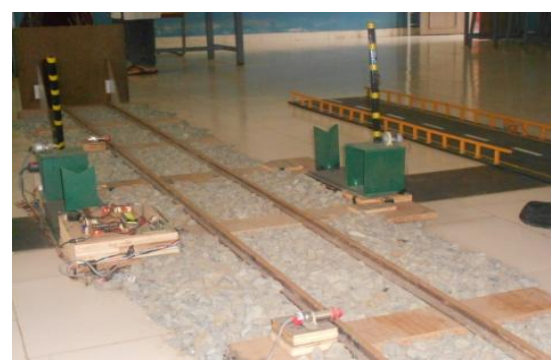

Figure 7: Gate fully open

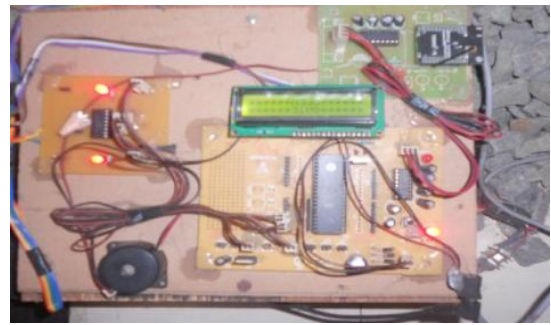

Figure 8: Gate controlling circuit

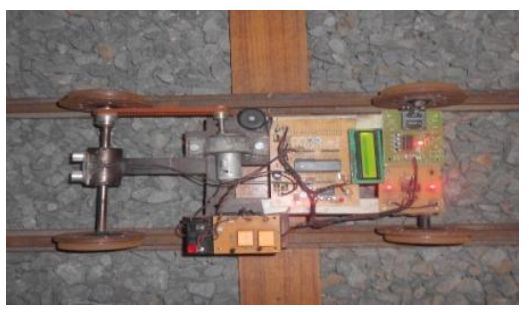

Figure 9: Developed model train

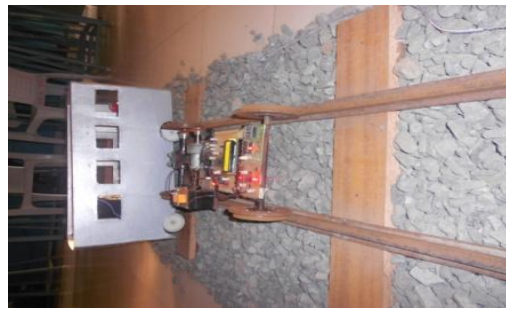

Figure 10: Model bus on the track

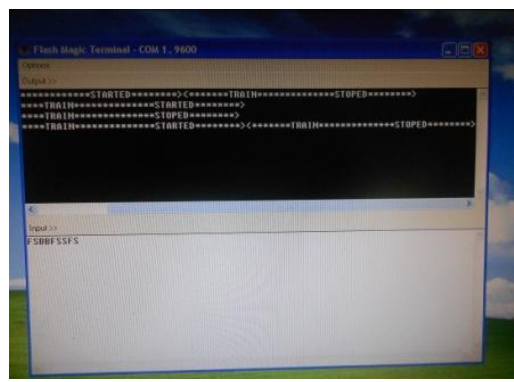

Figure 11: Signal information at control room 


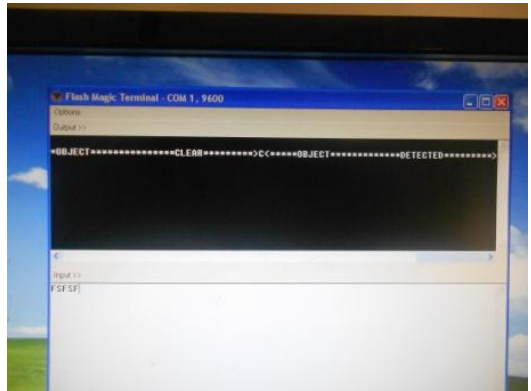

Figure 12: Signal information at control room

Figure 1and Figure 3 shows the train is away from the vibration sensor. The sensor draws an output signal ' 0 ' Volts results the gate is open. Figure 2, Figure 5, Figure 6 shows the train moving close to the rail gate results the gate is fully closed. Figure 8 shows the gate control circuit consisting of power section, controlling section, and IEEE 802.15.4, and display section. The signal from the vibration sensor is received at the input port of the microcontroller drive the motor assembly using L293D driver circuit, Causes to open and close the gate. The LCD shows the open state of the gate. Figure 10 shows an object i.e. model bus on the track results the stop position of the train to avoid the collision. Figure 11 and Figure 12 shows the results received at the control room. The start and stop position of the train and object detected status displayed on the flash magic terminal.

\section{CONCLUSION}

Safeguard the human life from miserable train accidents is having significant importance with the advancement of technology. The working model was fabricated within the institute premises. The results exhibit that it is one of the prudent approach for secure railway system. The ultrasonic sensor detect the target object in the detected zone in two seconds and communicate the control room is an added advantage of the proposed method. An alert message from the

sensor assembly transferred to the control room leads the train movements either to move forward or to stop. The communication between the control room and the train on the track will also an added advantage in this approach to safeguard from accidents. In future this developed working model is equipped with radar communication system to navigate the position of the train and the track. We are working to develop black box mechanism for recording the specific reason of accident for further analysis.

\section{ACKNOWLEDGMENTS}

The authors like to express sincere thanks to the management and principal of Sai Spurthi Institute of Technology for providing the fund and necessary infrastructure. And also thank to Mr. K. Krishna Prasad mechanical department for molding the trail wheels and gear sector arrangement.

\section{REFERENCES}

[1] Sandhya gautam, sandip nemade, teena sakla. simulation of an anti-collisionsystem on same track for railways. international journal of engineering and technology, vol. 2(9), page 4832-4837,2010.

[2] Ding, W. and Marchionini, G. 1997 A Study on Video Browsing Strategies. Technical Report. University of Maryland at College Park.

[3] Fröhlich, B. and Plate, J. 2000. The cubic mouse: a new device for three-dimensional input. In Proceedings of the SIGCHI Conference on Human Factors in Computing Systems.

[4] Tavel, P. 2007 Modeling and Simulation Design. AK Peters Ltd.

[5] Sannella, M. J. 1994 Constraint Satisfaction and Debugging for Interactive User Interfaces. Doctoral Thesis. UMI Order Number: UMI Order No. GAX9509398., University of Washington.

[6] Forman, G. 2003. An extensive empirical study of feature selection metrics for text classification. J. Mach Learn. Res. 3 (Mar. 2003), 1289-1305.

[7] Brown, L. D., Hua, H., and Gao, C. 2003. A widget framework for augmented interaction in SCAPE.

[8] Y.T. Yu, M.F. Lau, "A comparison of MC/DC, MUMCUT and several other coverage criteria for logical decisions", Journal of Systems and Software, 2005, in press.

[9] Spector, A. Z. 1989. Achieving application requirements. In Distributed Systems, S. Mullender

\section{AUTHOR BIOGRAPHY}

K.Vidyasagar: Received B. Tech degree in Instrument Technology from Andhra University College of Engineering Visakhapatnam, M.E from P.S.G.Tech Coimbator. He is now a research scholar under the guidance of Dr. A. Bhujangarao, Andhra University. His current research interests include image processing in biomedical instrumentation and related embedded systems.

P. Sekhar Babu received B.Tech degree in ECE from AZCET,JNTU, HYD.M.TECH from V.R.S.E.C ANU, Guntur. He is now a research scholar of JNTU Kakinada. His research interests in wireless communication \& Signal Processing. He published his research work in several conferences.

R. RamPrasad received his B.Tech degree in ECE from Sai Spurthi Institute of Technology, JNTU, Hyd. M.Tech From Nova Engineering College, JNTU, Kakinada.His research interest is on RF and Space Communication. He published several papers in various international conferences. 